\title{
Prefrontal Neuropsychological Predictors of Treatment Remission in Late-Life Depression
}

\author{
Guy G Potter', Joshua D Kittinger', H Ryan Wagner', David C Steffens' and K Ranga Rama Krishnan*,' \\ 'Department of Psychiatry and Behavioral Sciences, Duke University Medical Center, Durham, NC, USA
}

Recent studies suggest that neuropsychological measures involving the prefrontal cortex are associated with treatment remission in latelife depression. To further explore this issue, we studied the neuropsychological performance of I I 0 depressed individuals aged 60 years and over who are participating in an ongoing pharmacologic treatment study. Participants were clinically depressed at entry to the study as rated by the Montgomery-Asberg Depression Rating Scale (MADRS $\geqslant 15$ ), at which time they also completed a neuropsychological assessment that included measures of prefrontal/executive functions. A geriatric psychiatrist treating the participant using a standardized pharmacologic treatment algorithm evaluated the participant at baseline and 3-month follow-up, completing a MADRS at both visits. Using logistic discriminative procedures to predict depression remission at 3 months while controlling for age, gender, education, ethnicity, and baseline MADRS severity, we found that perseverative responses during verbal initiation tasks significantly predicted remission status (MADRS <7). This finding is consistent with previous single-agent treatment studies suggesting a relationship between prefrontal neuropsychological function and treatment response in late-life depression. The current results, however, appear to differentiate verbal perseveration from verbal initiation as the cognitive process that is most associated with poor treatment response. By extension, we suggest that orbitofrontal prefrontal cortex may play a role in sustaining perseverative processing in geriatric depression. Neuropsychopharmacology (2004) 29, 2266-227I, advance online publication, I September 2004; doi:I0.I038/sj.npp. I30055 I

Keywords: depression; elderly; prefrontal cortex; neuropsychological testing; treatment response

\section{INTRODUCTION}

While the involvement of prefrontal cortex in Major Depressive Disorder has been well documented (Mayberg et al, 1994; Bench et al, 1992), it is an especially salient issue in geriatric depression because this region of the brain is associated with disproportionate age-related decline ( $\mathrm{Raz}$ et al, 1997). In particular, MRI findings of deep white matter hyperintensities involving frontal-subcortical pathways are associated with both chronicity and cognitive impairment in late-life depression (Krishnan et al, 1998; Steffens et al, 2000; Lesser et al, 1996). These results are consistent with neurophysiological and neuropsychological data that associate prefrontal dysfunction with treatment resistance in depressed older adults (Alexopoulos et al, 2000; Kalayam and Alexopoulos, 1999), which is estimated to occur in up to one-third of this population (Schneider, 1996). As underlying deficits in prefrontal cortex appear to be associated with more intractable depression, assessment of prefrontal functions may improve treatment efficacy

\footnotetext{
* Correspondence: Dr KRR Krishnan, Department of Psychiatry and Behavioral Sciences, Duke University Medical Center Box 3950, Durham, NC 27710, USA, Tel: + I 919684 5156, Fax: + I 919 68I 5489, E-mail: krish00I@mc.duke.edu

Received I November 2003; revised II March 2004; accepted 12 March 2004
}

through early identification of individuals most likely to be refractory to routine clinical intervention.

Neuropsychological measures of prefrontal functions have potential utility as an efficient way to identify depressed individuals whose prefrontal deficits may predispose them to poor treatment outcome. Alexopoulos and co-workers found among a group of older depressed individuals enrolled in a pharmacologic treatment study that lower performance on a neuropsychological measure of prefrontal initiation/preservation (I/P) was associated with poorer acute treatment response (Kalayam and Alexopoulos, 1999) and higher rates of recurrence over a 2 -year follow-up period (Alexopoulos et al, 2000). The fact that they did not report the extent to which initiation and perseveration differentially contributed to their findings reflects the primary limitation of these studies, which is that the findings from a single brief measure are generalized to the multiple neurocognitive processes that are mediated by the prefrontal cortex. These processes are collectively referred to as executive functions, and include higher-order cognitive processes like attentional control, working memory, performance monitoring, and initiation (Lezak, 1995). As with the neuroanatomy of depression, executive functions are subserved by multiple pathways involving different regions of the prefrontal cortex (Cummings, 1995). Given the neuroanatomical heterogeneity of both depression and executive functions, it is important to 
examine whether treatment resistance in geriatric depression is associated with a focal neuropsychological process or whether it reflects a broader syndrome of prefrontal dysfunction that is detectable by other neuropsychological measures.

The purpose of the current study was to identify separable executive function processes that may be associated with treatment response in geriatric depression. One goal was to further examine the roles of initiation and perserveration as predictors of treatment response. In addition, we sought to identify the role of other executive processes, including attentional control, working memory, and performance monitoring, that may be associated with treatment response in geriatric depression.

\section{PATIENTS AND METHODS}

\section{Sample}

Participants consisted of 110 depressed individuals enrolled in the NIMH-sponsored Mental Health Clinical Research Center (MHCRC) at Duke University Medical Center (DUMC). All participants diagnosed as depressed met the DSM-IV criteria for Major Depressive Disorder at entry to the study and were at least 60 years of age. The exclusion criteria for the MHCRC study were: (1) another major psychiatric illness (anxiety is considered acceptable if secondary to depression); (2) alcohol or drug abuse or dependence; (3) clinically diagnosed primary neurological condition (eg Parkinson's disease, stroke), including dementia as addressed below; and (4) nonpsychotropic medications, medical illness, or physical disability that may affect cognitive function. Prior to entering the study, all prospective participants received an explanation of its purpose and of procedures that would be involved. Individuals who provided written informed consent were subsequently enrolled.

\section{Treatment Protocol}

All participants in the study were followed by a geriatric psychiatrist who provided treatment based on the Duke STAGED approach (Steffens et al, 2002), which follows a standardized treatment algorithm. This algorithm simulates clinical treatment practices as opposed to clinical trial design by weighing past treatment and current severity in the decision-making process. Participants without prior treatment are initially prescribed a selective serotonin reuptake inhibitor (SSRI). If treatment with an SSRI does not produce sufficient response after 8 to 12 weeks, the next option is to either change treatment to venlafaxine or to augment with bupropion. If these changes do not result in an adequate treatment response, further options include tricyclic antidepressants or lithium augmentation. Each treatment medication that is introduced is increased as recommended up to the maximum approved dose or as tolerated by the participant. In the current sample, 68 participants received a single treatment agent (50 SSRI, eight venlafaxine, five bupropion, two nefazodone, two mirtazapine, one phenelzine). Treatment with consecutive single agents occurred among five participants, while 34 participants were treated with a combined-agent approach.
Pharmacologic treatment was not completed for three participants during the study period.

In the STAGED approach, electroconvulsive therapy is a treatment option at each level of the algorithm based on depression severity, previous failed trials, and participant preference. In the current cohort, only four participants received ECT during the time period that was studied. A small minority of participants were referred for psychotherapy, while a minority of others were involved in psychotherapy at entry to the study.

Study investigators monitored treatment to ensure that the clinical protocol was properly followed. Participants were evaluated every 3 months, with more frequent followup when clinically indicated.

\section{Assessment Procedures}

At baseline, participants received standardized clinical assessments, including the Clinical Global Impression Scale (Guy, 1976), the Hamilton Rating Scale for Depression (Hamilton, 1960), the Duke Depression Evaluation Schedule (Landerman et al, 1989), and the Montgomery-Asberg Depression Rating Scale (MADRS; Montgomery and Asberg, 1979). The MADRS is a 10-item scale of depression severity that is based on patient report and clinical observation. Scores on the MADRS range from 0 to 60 , with higher scores indicating greater severity of depressive symptomatology. The MADRS was completed by the treating psychiatrist at entry to the study and at each interval visit. In the current study, we used the MADRS score to track treatment response and define remission.

Baseline cognitive screen. Participants were excluded from the study if they had dementia or suspected dementia at baseline. For each participant, MHCRC geriatric psychiatrists administered a clinical evaluation, reviewed medical records, and conferred with referring physicians. All participants completed the Mini-Mental State Examination (MMSE; Folstein et al, 1975). Some severely depressed individuals scored below 25 on the MMSE, which is often used as a cutoff score for suspected dementia. The protocol for the MHCRC study in such cases is to follow these participants through an acute 8-week phase of treatment to determine if cognition improves. Individuals whose MMSE scores remain below 25 are not followed longitudinally by the study.

Neuropsychological assessment. A neuropsychological assessment was administered to all participants at or near the time of study enrollment. The full assessment is based on a battery that was initially developed to assess cognitive impairments associated with Alzheimer's disease and related disorders, but has been shown to be sensitive to neuropsychological deficits in depression as well (Potter et al, 2003). In keeping with the research aims of this study, only measures of executive function were used. Attentional control was represented by the Trail Making Test (Parts A and B; Reitan, 1992). Verbal initiation for lexical information was assessed by the Controlled Oral Word Association Test (COWA) from the Multilingual Aphasia Examination (Benton et al, 1983) and initiation for semantic information was assessed by a category fluency task (Animal Naming). 
Perseveration was assessed by four measures: (1) perseverative errors committed on the Benton Visual Retention Test (BVRT, Benton, 1974), (2) perseverative errors on COWA, (3) perseverative errors on Animal Naming, and (4) the sum of perseverative errors committed across the COWA and Animal Naming tasks. The latter measure was included to improve the distribution of responses over that of the individual measures. Performance monitoring was assessed by summing the number of confabulatory errors occurring across the four trials of a word-list memory task that was part of the assessment battery. Simple auditory working memory was represented by the forward trial of WAIS-R Digit Span (Wechsler, 1987). Complex auditory working memory was assessed by the backward trial of the Digit Span task.

\section{Analytic Strategy}

Neuropsychological performance and depressive symptomatology (MADRS) were assessed at entry to the treatment study. The MADRS was also assessed 3 months after entry to the study. The analytical cohort was restricted to individuals with a baseline MADRS score of 15 or greater in order to exclude individuals whose scores were more consistent with subclinical depression.

A dichotomous outcome variable denoting remission was coded positive for participants whose 3-month MADRS score was less than seven. Using the aforementioned neuropsychological measures, a set of 11 unidirectional planned comparisons were estimated to examine the association between baseline neuropsychological function and subsequent treatment response. For seven of the measures, low values were predicted to be associated with greater odds of remission at follow-up: (1) COWA perseveration, (2) Animal Naming perseveration, (3) combined COWA and Animal Naming perseveration, (4) BVRT perseverative errors, (5) verbal confabulation, (6) Trail Making A, and (7) Trail Making B. For the remaining four measures, higher values were predicted to be associated with greater odds of remission: (1) Digit Span forward, (2) Digit Span backward, (3) COWA, and (4) Animal Naming.

Bivariate differences between individuals meeting the MADRS remission criteria at follow-up and nonremitting individuals were tested using Student's $t$-tests; nonparametric Wilcoxon's tests were used where distributional assumptions of normality failed. Multivariable modeling was subsequently conducted by regressing the MADRS remission variable on the individual neuropsychological measures using logistic regression procedures. Each measure was modeled separately, and all models included covariates for age, gender, race, education, and the baseline MADRS score. Measures of verbal perseveration included an additional covariate to account for the number of perseverative errors relative to the total number of correct responses produced.

\section{RESULTS}

The demographic characteristics of the cohort are described in Table 1. Individuals in remission at 3-month follow-up did not differ significantly at baseline from nonremitted individuals on any dimension, including the baseline depression measurements (MADRS).

Bivariate tests of differences between remitting and nonremitting individuals are presented in Table 2. Individuals in remission at 3-month follow-up made significantly fewer perseverative errors on the COWA task relative to the nonremitters and were significantly higher at baseline on Digit Span forward. The number of perseverative errors derived from combined COWA and Animal Naming tasks was lower among remitted individuals and approached statistical significance. Perseverative errors on the Animal Naming task were also substantially lower (47\%), although the difference was not statistically significant. No other differences attained significance.

Results from multivariable analyses were similar (Table 3). Odds of remission decreased by approximately $26 \%$ for each additional perseverative error using combined ratings from the COWA and Animal Fluency tasks. Perseverative errors on the two constituent tasks - COWA and Animal Naming-were also associated with decreased odds of remission, with effects approaching significance. Odds of

Table I Sample Characteristics

\begin{tabular}{lccc}
\hline & $\begin{array}{c}\text { Complete } \\
\text { sample } \\
(\mathbf{n}=\mathbf{I} \mathbf{~ I 0 )}\end{array}$ & $\begin{array}{c}\text { Nonremitted } \\
(\mathbf{n}=\mathbf{8 7})\end{array}$ & $\begin{array}{c}\text { Remitted } \\
(\mathbf{n}=\mathbf{2 3})\end{array}$ \\
\hline Age (years), mean (SE) & $73.78(0.74)$ & $73.76(0.80)$ & $73.87(1.90)$ \\
\% Female & $59.09 \%$ & $58.62 \%$ & $60.87 \%$ \\
\% White & $89.09 \%$ & $90.80 \%$ & $82.61 \%$ \\
Education (years), mean (SE) & $|3.6|(0.85)$ & $14.00(0.38)$ & $13.65(0.94)$ \\
Baseline MADRS, mean (SE) & $24.65(0.62)$ & $24.74(0.67)$ & $24.35(1.55)$ \\
\hline
\end{tabular}

Table 2 Bivariate Analysis of Neuropsychological Measures by Remission Status

\begin{tabular}{|c|c|c|c|}
\hline & $\begin{array}{c}\text { Nonremitted, } \\
\text { mean (SE) } \\
(n=87)\end{array}$ & $\begin{array}{c}\text { Remitted, } \\
\text { mean (SE) } \\
(n=23)\end{array}$ & $P$ \\
\hline Digit span_-forward & $8.27(0.27)$ & $9.64(0.55)$ & 0.046 \\
\hline Digit span-backward & $6.73(0.26)$ & $7.14(0.65)$ & NS \\
\hline COWA — total correct & $37.35(1.19)$ & $36.35(1.94)$ & NS \\
\hline $\begin{array}{l}\text { Animal naming }(A N) \text { - total } \\
\text { correct }\end{array}$ & |5.0| (0.48) & I5.48 (1.18) & NS \\
\hline BVRT_-perseverative errors & $1.03(0.15)$ & $1.30(0.26)$ & NS \\
\hline COWA - perseverative errors & $1.43(0.18)$ & $0.78(0.23)$ & 0.048 \\
\hline AN_-perseverative errors & $0.55(0.12)$ & $0.30(0.12)$ & NS \\
\hline $\begin{array}{l}\text { Total perseverative errors } \\
(C O W A+A N)\end{array}$ & $2.17(0.25)$ & $1.27(0.32)$ & 0.059 \\
\hline Confabulations & $0.97(0.15)$ & $0.87(0.30)$ & NS \\
\hline Trail making A (sec) & $56.62(5.52)$ & $61.61(10.12)$ & NS \\
\hline Trail making B (sec) & | $44.92(9.11)$ & $164.52(22.00)$ & NS \\
\hline
\end{tabular}

NS = not significant. 
Table 3 Odds Ratios and Confidence Intervals for Neuropsychological Measures

\begin{tabular}{lccc}
\hline & $\begin{array}{c}\text { Odds } \\
\text { ratio }\end{array}$ & $\mathbf{9 5 \%} \mathbf{~ C l}$ & $\mathbf{P}$ \\
\hline Digit span_forward & 1.31 & $0.99: 1.72$ & 0.056 \\
Digit span_backward & 1.11 & $0.83: 1.47$ & NS \\
COWA_total correct & 0.99 & $0.95: 1.04$ & NS \\
Animal naming (AN)—total correct & 1.06 & $0.93: 1.19$ & NS \\
BVRT_perseverative errors & 1.12 & $0.79: 1.57$ & NS \\
COWA_perseverative errors & 0.71 & $0.49: 1.05$ & 0.082 \\
AN_perseverative errors & 0.56 & $0.29: 1.05$ & 0.079 \\
Total perseverative errors (COWA+AN) & 0.74 & $0.55: 0.99$ & 0.045 \\
Confabulations & 0.95 & $0.67: 1.35$ & NS \\
Trail Making A (sec) & 1.00 & $0.99: 1.01$ & NS \\
Trail Making B (sec) & 1.00 & $0.99: 1.01$ & NS \\
\hline
\end{tabular}

NS $=$ not significant.

remission increased 1.3 times for each unit increment on Digit Span forward; however, unlike the significant biserial correlation, this result was only marginally significant. None of the remaining neuropsychological measures were significantly associated with remission in either bivariate or multivariable tests.

\section{DISCUSSION}

The primary finding of this study is that an index of verbal perseveration among depressed older adults was associated with a lower remission rate after 3 months of pharmacologic treatment using a standardized algorithmic approach. For the combined verbal perseveration measure, each additional perseverative error lowered odds of remission by $26 \%$. This result was found after controlling for age, gender, ethnicity, education, and baseline MADRS, which suggests that it is robust across demographic differences and baseline depression severity.

Our results add to previous findings of an association between prefrontal executive functions and treatment response in geriatric depression. Unlike previous treatment trial studies using a single pharmacologic agent (Kalayam and Alexopoulos, 1999), the current data reflect optimal treatment over a period of 3 months. We believe that the strength of this study is that it complements previous research based on treatment response to a single agent.

With regard to Kalayam and Alexopoulos' (1999) report that lower scores on the I/P scale from the Mattis Dementia Rating Scale (Mattis, 1989) were associated with poor treatment response, we found evidence for an effect of verbal perseveration, but not of verbal initiation or visual memory perseveration. These findings may add some clarity to the role of perseveration $v s$ initiation as a predictor of treatment resistance, but are limited by the fact that different measures were used to assess the constructs of initiation and perseveration between the two studies. Although the Animal Naming task is comparable to the semantic fluency task used on the I/P scale, the I/P scale includes an additional - albeit less complex - task requiring individuals to name items worn by the test administrator. In addition, the perseveration items on this measure involve primarily motor and visuomotor tasks. There were also differences in treatment length (6 vs 12 weeks) and definition of remittance (Cornell Scale $<7 v s$ MADRS $<7$ ). On the whole, however, the methodologies are similar. Factor analysis of the Mattis I/P scale in an older treatment-resistant population may shed further light on the relative contributions of initiation and perseveration in predicting response to pharmacologic treatment of depression.

Our results with regard to perseveration are also consistent with a study of middle-aged depressives finding that perseverative responses on the Wisconsin Card Sorting Test were associated with a better treatment response to fluoxetine (Dunkin et al, 2000). Although that study is limited by a small sample size $(n=14)$, it does suggest that perseverative tendencies contribute to poor treatment across a broad age spectrum. These results, in addition to those of the current study and others with geriatric samples (Kalayam and Alexopoulos, 1999; Alexopoulos et al, 2000), suggest that further research is needed to identify the types of perseveration that are associated with poor treatment response, with particular attention to age effects.

Theoretically, our results are consistent with data from multiple sources that implicate the prefrontal cortex in Major Depressive Disorder. Perseverative tendencies have long been associated with deficits in prefrontal cortex, particularly in the orbitofrontal region (Joseph, 1999), and it has been argued that hypoactivation in this region undermines the capacity to over-ride the repetitive cycle of negative affect and dysfunctional cognitions that accompanies depression (Davidson et al, 2002). In other words, orbitofrontal hypoactivation may foster the perseverative negative thinking that sustains depressed affect.

The positive association between Digit Span forward and treatment remission that was found in the biserial correlation is to our knowledge unique in the literature on treatment response in late-life depression. Relative to perseveration, this suggests an additional area of the prefrontal cortex that may contribute to treatment response in this population. Some researchers have proposed that Digit Span forward assesses the efficiency of attentional processes, including the ability to screen out distraction (Lezak, 1995; Kaufman et al, 1991). In the context of depression, a deficit in Digit Span forward may correlate with functional difficulties in maintaining mental set in the face of distracting affective input. Replicative studies of Digit Span forward are needed to better determine the role that this measure may play in treatment response among older adults.

The clinical implication of our findings is that measures assessing verbal perseveration and simple auditory working memory may be useful in identifying individuals whose neuropsychological deficits in these areas predispose them to greater treatment difficulty. The significance of this is less that these measures are clinically sensitive to treatment resistance in and of themselves, but more that they can aid in characterizing a profile of performance deficits detectable by neuropsychological testing. In this regard, we note that 
mean scores on some putative measures of executive function were slightly better among the nonremitted group, albeit not in terms of statistical significance. This suggests that neuropsychological predictors of pharmacologic treatment response may be localized to specific prefrontal processes like verbal perseveration, rather than generalized to a more global dysexecutive syndrome. Given our results, it may be that this locus involves the orbitofrontal region of prefrontal cortex.

If individuals with prefrontal deficits can eventually be reliably identified as at-risk for poor treatment outcome, one response may be to implement a more proactive treatment plan, with more frequent monitoring of symptomatology, greater attention to functional disabilities, and multimodal treatment including electroconvulsive therapy and psychotherapy. Relevant to this, one study found that a 12 -week treatment of problem-solving therapy was associated with higher remission rates and lower disability among depressed elders with executive dysfunction compared to individuals who were treated with supportive therapy (Alexopoulos et al, 2003). The authors argued that part of this effect was due to the fact that the problemsolving intervention offered alternative behavioral strategies to individuals who were unable to stop repeating their maladaptive responses due to compromised prefrontal function. The therapy was conducted in the absence of pharmacotherapy, and we presume that clinical efficacy would be further enhanced by a combined psychotherapy/ pharmacotherapy intervention.

One limitation of the current study is that treatment for depression was not strictly controlled. As our treatment algorithm included individual factors in the decision process, there was variation in the types and duration of pharmacologic agents administered to participants, as well as occasional differences in nonpharmacological treatment. This greater variability among participants may have resulted in additional error variance and also limits our ability to relate our results to a specific neuropharmacologic agent or mechanism. On the other hand, because this algorithm attempts to replicate a naturalistic treatment approach, it may be more representative of real-world outcomes than results based on treatments modeled after clinical trials. Yet, even a naturalistic algorithm does not adequately reflect the manner in which most depressed older adults are treated in the community, which may be best approached by large community-based studies.

Another limitation of the current study is the lack of a neuropsychological assessment at follow-up. As a result, we do not know whether neuropsychological performance improved with remission, which would help clarify whether executive dysfunction is an etiology of depression or whether it is reversible with successful treatment.

In summary, the current study found that prefrontal deficits characterized by higher rates of verbal perseveration and poorer simple auditory working memory span were associated with a lower rate of treatment remission in older depressed adults over a 3-month period of pharmacologic treatment. Future studies should continue to examine the contribution of specific prefrontal neuropsychological processes to late-life depression, with particular attention to long-term remission and the prevention of adverse outcomes.

\section{ACKNOWLEDGEMENTS}

We do not have any affiliations that could potentially bias this work. We gratefully acknowledge Karee Powers for her assistance with data coding and management, and Kathleen Welsh-Bohmer, $\mathrm{PhD}$, for her consultation on scientific and technical issues. This study was supported in part by the Conte Center for the Study of Depression in Late Life, including NIMH Grants P50 (MH60451) and R01 (MH54846).

\section{REFERENCES}

Alexopoulos GS, Meyers BS, Young RC, Kalayam B, Kakuma T, Gabrielle $M$ et al (2000). Executive dysfunction and long-term outcomes of geriatric depression. Arch Gen Psychiatry 57: 285290.

Alexopoulos GS, Raue P, Arean P (2003). Problem-solving therapy versus supportive therapy in geriatric major depression with executive dysfunction. Am J Geriatr Psychiatry 11: 46-52.

Bench CJ, Friston KJ, Brown RG, Scott LC, Frackowiak RS, Dolan RJ (1992). The anatomy of melancholia: focal abnormalities of cerebral blood flow in major depression. Psychol Med 22: 607-615.

Benton A (1974). Revised Visual Retention Test: Clinical and Experimental Applications, 4th edn. Psychological Corporation: New York.

Benton AL, Hamsher K, Varney N, Spreen O (1983). Contributions to Neuropsychological Assessment. Oxford University Press: New York.

Cummings JL (1995). Anatomic and behavioral aspects of frontalsubcortical circuits. Ann NY Acad Sci 769: 1-13.

Davidson RJ, Pizzagalli D, Nitschke JB, Putnam K (2002). Depression: perspectives from affective neuroscience. Annu Rev Psychol 53: 545-574.

Dunkin JJ, Leuchter AF, Cook IA, Kasl-Godley JE, Abrams M, Rosenberg-Thompson S (2000). Executive dysfunction predicts nonresponse to fluoxetine in major depression. J Affect Disord 60: 13-23.

Folstein MF, Folstein SE, McHugh PR (1975). Mini-mental state. A practical method for grading the cognitive state of patients for the clinician. J Psychiatr Res 12: 189-198.

Guy W (1976). ECDEU Assessment Manual for Psychopharmacology, (DHEW Publication ADM 76-338). National Institute of Mental Health, Psychopharmacology Research Branch: Rockville, MD.

Hamilton M (1960). A rating scale for depression. J Neurol Neurosurg Psychiatry 23: 55-61.

Joseph R (1999). Frontal lobe psychopathology: mania, depression, confabulation, catatonia, perserveration, obsessive compulsions, and schizophrenia. Psychiatry 62: 138-172.

Kalayam B, Alexopoulos GS (1999). Prefrontal dysfunction and treatment response in geriatric depression. Arch Gen Psychiatry 56: 713-718.

Kaufman AS, McLean J, Reynolds C (1991). Analysis of WAIS-R factor patterns by sex and race. J Clin Psychol 47: 548-557.

Krishnan KR, Hays JC, George LK, Blazer DG (1998). Six-month outcomes for MRI-related vascular depression. Depress Anxiety 8: $142-146$.

Landerman R, George LK, Campbell RT, Blazer DG (1989). Alternative models of the stress buffering hypothesis. Am J Community Psychol 17: 626-642.

Lesser IM, Boone KB, Mehringer CM, Wohl MA, Miller BL, Berman NG (1996). Cognition and white matter hyperintensities in older depressed patients. Am J Psychiatry 153: 1280-1287.

Lezak MD (1995). Neuropsychological Assessment, 3rd edn. Oxford University Press: New York. 
Mattis S (1989). Dementia Rating Scale. Psychological Assessment Resources: Odessa, FL.

Mayberg HS, Lewis PJ, Regenold W, Wagner HN (1994). Paralimbic hypoperfusion in unipolar depression. J Nucl Med 35: 929-934.

Montgomery SA, Asberg M (1979). A new depression scale designed to be sensitive to change. Br J Psychiatry 134: 382-389.

Potter GG, Welsh-Bohmer KA, Wagner HR, Steffens DC (2003). Detection and staging of cognitive impairment in geriatric depression. J Int Neuropsychol Soc 9: 275 (abstract).

Raz N, Gunning FM, Head D, Dupuis JH, McQuain J, Briggs SD et al (1997). Selective aging of the human cerebral cortex in vivo: Differential vulnerability of the prefrontal gray matter. Cereb Cortex 7: 268-282.
Reitan RM (1992). Trail Making Test: Manual for Administration and Scoring. Reitan Neuropsychological Laboratory: Tuscon, AZ.

Schneider LS (1996). Pharmacologic considerations in the treatment of late-life depression. Am J Geriatric Psychiatry 4(Suppl 1): S51-S65.

Steffens DC, MacFall JR, Payne ME, Welsh-Bohmer KA, Krishnan KR (2000). Grey-matter lesions and dementia. Lancet 356: $1686-1687$.

Steffens DC, McQuoid DR, Krishnan KRR (2002). The Duke Somatic Treatment Algorithm for Geriatric Depression (STAGED) approach. Psychopharmacol Bull 36: 58-68.

Wechsler D (1987). Wechsler Memory Scale-Revised. The Psychological Corporation: San Antonio, TX. 